\title{
Philosophiques
}

\section{Otfried Höffe, Principes du droit, (traduction par Jean-Christophe Merle), Paris, Les Éditions du Cerf, 1993.}

\section{Daniel M. Weinstock}

Volume 23, numéro 1, printemps 1996

Critères esthétiques et métamorphoses du beau

URI : https://id.erudit.org/iderudit/027387ar

DOI : https://doi.org/10.7202/027387ar

Aller au sommaire du numéro

Éditeur(s)

Société de philosophie du Québec

ISSN

0316-2923 (imprimé)

1492-1391 (numérique)

Découvrir la revue

Citer ce compte rendu

Weinstock, D. M. (1996). Compte rendu de [Otfried Höffe, Principes du droit, (traduction par Jean-Christophe Merle), Paris, Les Éditions du Cerf, 1993.]

Philosophiques, 23(1), 196-198. https://doi.org/10.7202/027387ar d'utilisation que vous pouvez consulter en ligne.

https://apropos.erudit.org/fr/usagers/politique-dutilisation/ 
Otfried Höffe, Principes du droit, (traduction par Jean-Christophe Merle), Paris, Les Éditions du Cerf, 1993.

Ce livre se donne comme but de démontrer l'actualité de la pensée d'Emmanuel Kant pour l'élaboration d'une philosophie du droit à mème de répondre aux importants défis moraux et politiques de la modernité. Les principales alternatives théoriques auxquelles Höffe croit devoir répondre au nom d'une théorie kantienne sont, d'une part, les différentes formes de lirrationalisme post-modeme qui rejettent toute prétention rationaliste, et qui, du moins dans certaines de ses manifestations, préfèrent s'en remettre à une forme de phronesis néo-aristotélicienne qui serait, croit-on, particulièrement appropriée au contexte de pluralité et de diversification sociale caractéristique des sociétés occidentales contemporaines, et, d'autre part, les théories sociales " empiro-pragmatiques ", notamment l'utilitarisme, qui estiment pouvoir tirer les outils nécessaires à l'élaboration d'une théorie du droit de la conception de la rationalité instrumentale à la base des sciences sociales et économiques.

Le livre est divisé en trois parties comptant chacune quatre chapitres. La première partie est dévouée à la fondation des principes du droit dans la pensée de Kant. Elle insiste sur la racine principielle commune qui unit au plus haut niveau d'abstraction l'éthique et la philosophie du droit de Kant, et met l'accent sur la place centrale qu'occupe dans la pensée kantienne la notion de droit individuel, primoridiale selon Höffe pour toute théorie juridique valable, et dont les théories strictement empiriques ne parviennent pas à rendre compte de manière adéquate.

L'intérêt principal de cette partie du livre réside à mon avis dans la tentative à laquelle se livre Höffe de désamorcer les sempitemelles critiques adressées à la théorie morale kantienne, selon lesquelles elle serait excessivement abstraite et désincarnée. Höffe fait valoir que l'êthique kantienne n'est pas métaphysique dans un sens qui serait ontologiquement ou épistémologiquement onéreux ; elle ne l'est qu'au sens où "son objet, la morale juridique, transcende tout motif empirique * (p. 71). Et il fait également remarquer, à 
juste titre, que ce n'est que dans sa forme la plus générale que l'impératif catégorique kantien prend cette forme abstraite que lui ont reprochée de multiples commentateurs depuis Herder et Hegel : Kant ne dérive des principes moraux et juridiques qu'après leur avoir fait suivre la médiation de données anthropologiques sur l'être humain et sur sa condition.

Höffe participe donc par ces arguments à ce courant des ètudes kantiennes qui cherche à faire valoir la compatibilité des principales doctrines du sage de Königsberg avec les théories philosophiques contemporaines portant sur la mètaphysique des valeurs ainsi que sur l'anthropologie philosophique. On regrettera simplement que Höffe ne semble pas vouloir complètement abandonner l'interprétation ontologique de la notion kantienne du "monde intelligible *, et surtout, que ses lectures de Kant planent souvent à une certaine distance du texte, en ce sens qu'il ne se livre jamais réellement à une reconstruction serrée des arguments de Kant qui chercherait à montrer le bienfondé d'une lecture * anthropologique " de la morale kantienne et à révéler la validité des arguments que cette lecture permet de faire ressortir des textes kantiens. Le lecteur qui veut s'assurer de la plausibilité d'une lecture anthropologique de Kant sera dans cette optique mieux servi par les exégètes anglo-saxons de Kant, dont, notamment, Henry Allison, Onora O'Neill, Barbara Herman et Thomas Hill.

La deuxième partie quitte le domaine méta-éthique pour discuter de l'impact de la théorie kantienne au niveau des théories éthiques de premier ordre. On y retrouve une critique kantienne de la tentative par John Stuart Mill de rendre compte des droits individuels et de la justice sociale distributive de l'intérieur de la théorie utilitariste. Les arguments de Höffe contre cette tentative de réconciliation sont pour l'essentiel probants. Mais il y a lieu de se demander dans quelle mesure on peut vraiment y voir une démonstration convaincante du fait que l'utilitarisme est irrémédiablement incapable de faire une place convenable aux notions de droit. En effet, le siècle qui a suivi la publication de L'Utilitarisme de John Stuart Mill a vu une prolifération d'œuvres dévouées à la reformulation du projet utilitariste. Ceux-ci (je pense ici notamment aux travaux de David Brink et de Peter Railton) prennent en ligne de compte un bon nombre des objections que Höffe adresse à Mill, et développent des théories qui sont ainsi autrement plus sophistiquées que celle de ce dernier. L'intérêt de ce chapitre est par ailleurs également diminué par le fait que Höffe ne tient pas compte de la littérature critique récente sur Mill, qui nous a fait découvrir des ressources insoupçonnees dans le corpus millien pour répondre à certaines des objections ennoncées ici.

Cette section du livre contient également des chapitres sur la doctrine kantienne de l'interdiction de la fausse promesse, oủ Höffe signale la distinction. tout à fait essentielle pour la compréhension de Kant, entre le conflit de devoirs et le conflit entre devoir et inclination, ainsi que sur la doctrine pénale de Kant. qui met l'accent sur la réparation, et que Höffe défend de manière convaincante contre les théories de la peine juridique mettant l'accent sur la dissuasion ou la réforme, et un chapitre sur le droit international, qui expose clairement l'essai de Kant sur la paix perpétuelle sans apporter grand-chose à son interprétation.

La dernière partie du livre traite des écrits de néo-kantiens contemporains œuvrant dans le domaine de l'éthique et de la philosophie politique: John Rawls, Karl-Otto Apel et Jürgen Habermas. (On y retrouve également un essai quelque peu incongru sur la théorie évolutionniste de Robert Axelrod). Il s'agit à bien des égards de la partie la plus intéressante du livre. Höffe reproche à Rawls l'utilisation que fait ce dernier de la theorie instrumentale de la rationalité, et dont il pare ses participants hypothétiques à la " position 
originelle *. Höffe a raison de noter que ce n'est que par l'ajout de prémisses morales quelque peu gratuites que Rawls réussit à retrouver au terme de son argumentation les droits individuels qu'il cherchait à fonder par la raison, et à éviter la position de l'utilité moyenne maximale, que John Harsanyi avait du reste dérivée d'une expérience de la pensée similaire à celle de Rawls. (Rawls huimême a rapidement compris que la rationalité "économique * et la théorie de la décision classique servaient bien mal son projet, et les a explicitement abandonnées peu de temps après la publication de la Théorie de la justice.) Höffe a également des choses interressantes à dire sur le rapport entre les deux célèbres principes de la justice sociale de Rawls : il suggère que le * principe de la différence * assurant une certaine redistribution matérielle envers les plus démunis aurait intérèt à ètre dérivé non directement de la position originelle, mais plutōt comme assurant la pleine application des droits juridiques et politiques garantis par le premier principe. Il s'agit là d'une suggestion intéressante qui mériterait d'être développée.

Les articles sur Apel et Habermas sont également dignes de mention. Höffe montre que le pas " au-delà " de Kant que Apel estime avoir fait en rejetant le paradigme de la conscience individuelle pour la perspective intersubjective de la communication n'a de réel impact sur la théorie éthique que si la " communication " dont on part est " moralisée " par l'introduction de normes et de contraintes morales qui présupposent justement l'éthique kantienne qu'on espérait dépasser. Et contre Habermas, il insiste sur le fait que sa "critique de la raison fonctionaliste "qui espère redonner à certaines sphères de l'activité humaine leur intégrité non-fonctionaliste et non-instrumentale présuppose une vision romantique, mais fort peu plausible d'un passé dans lequel l'intégrité de ces sphères aurait été respectée.

Dans l'ensemble, il s'agit donc d'un livre assez inégal. On y trouve des textes très intéressants, mais également des contributions d'intérêt minime pour quiconque a suivi les débats philosophiques récents. On regrettera également tout au long du livre une certaine inattention aux textes étudiés: Höffe commente souvent à une trop grande distance des textes. De manière générale, la prose aurait gagné à ètre quelque peu retravaillée, car elle pèche parfois par excès d'obscurité. Il m'est impossible de déterminer s'il s'agit là de la faute du traducteur ou de l'auteur lui-même.

Daniel M. Weinstock

Département de philosophie

Université de Montréal 